\title{
Validación para Colombia del índice RESVECH 2.0 valoración de cicatrización en heridas crónicas
}

para la

\author{
valoracion de cicatrización en heridas cronicas
}

\section{Validation for Colombia of the RESVECH 2.0 index for the assessment of wound healing in chronic wounds}

\author{
Juan Carlos Restrepo Medrano ${ }^{1}$ \\ Recibido para publicación: Octubre 18 de 2018 - Aprobado para publicación: Mayo 15 de 2019
}

\begin{abstract}
RESUMEN
Objetivo. Validar en Colombia el índice RESVECH 2.0 para valorar y evaluar el proceso de cicatrización en heridas crónicas. Materiales y métodos. Estudio de validación realizado en tres hospitales de III - IV nivel de complejidad durante los años 2013 y 2016. Población: 120 pacientes con alguna de las lesiones incluidas en el estudio Estadísticamente se utilizaron la prueba de esfericidad de Barlett, la prueba de adecuación de la muestra de KaiserMeyer-Olkin, el Coeficiente alfa de Cronbach y la Confiabilidad test-test. Resultados. el índice de medida RESVECH 2.0 es un instrumento que permite la valoración de las heridas crónicas a pie de cama de manera sencilla y que cuenta con adecuadas medidas psicométricas. Conclusiones. El índice RESVECH 2.0 cuenta con alta fiabilidad y validez, por lo que se recomienda usarlo en guías clínicas de cuidado de pacientes con heridas crónicas en población colombiana.
\end{abstract}

Palabras clave: validación, valoración, cicatrización, heridas crónicas.

\begin{abstract}
Objective. To validate in Colombia the RESVECH 2.0 index to assess and evaluate the healing process in chronic wounds. Materials and methods. Validation study carried out in three hospitals of III - IV complexity level of attention between 2013 and 2016. Population: 120 patients with any of the injuries included in the study Statistically the sphericity test of Barlett was used, the adequacy test of the KaiserMeyerOlkin sample, the Cronbach alpha coefficient and the Reliability test-test. Results. The RESVECH 2.0 measurement index is an instrument that allows the assessment of chronic wounds at the foot of bed in a simple way and has adequate psychometric measures. Conclusions. The RESVECH 2.0 index has high reliability and validity, reasons to be recommended to use it in clinical guidelines for the care of patients with chronic wounds.
\end{abstract}

Keywords: Validation, assessment, healing, chronic wounds
Citación (Vancouver)

Restrepo Medrano. Validación para Colombia del índice RESVECH 2.0 para la valoración de cicatrización en heridas crónicas. Rev Avances en Salud; 2019. (3)1:8-15 . DOI: $\underline{10.21897 / 25394622.1748}$
@ 2019. Universidad de Cordoba. Este es un artículo de acceso abierto distribuido bajo los términos de la licencia Creative Commons Attribution License, que permite el uso ilimitado, distribución y reproducción en cualquier medio, siempre que el autor original y la fuente se acreditan.

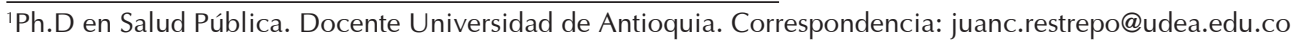




\section{INTRODUCCIÓN}

Las heridas crónicas se han convertido en un importante problema de salud (1), debido a sus implicaciones tanto para pacientes y familiares, como para los servicios de salud, donde se observa aumento desmedido de costos en la atención y en ocasiones una disminución de la calidad asistencial en estos pacientes (2), ya que entre muchos factores no contamos con instrumentos válidos y fiables que nos permitan monitorear los diferentes procesos en la atención de estos pacientes.

Las cifras de prevalencia de heridas crónicas, que se encuentran en la bibliografía, son variables y dependen de la población y los servicios estudiados, al punto que pueden oscilar entre el $2 \%$ y el $50 \%$, siendo mayores en las unidades de medicina interna o larga estancia $(3,4,5)$. Todo lo anterior afecta de forma directa la calidad asistencial en estos pacientes, la cual se define como el grado en que la atención prestada se ajusta a los criterios y estándares basados en la evidencia científica, poniendo de manifiesto el nivel óptimo de asistencia (6); este concepto juega un papel muy importante y depende de factores tan diversos como los conocimientos científicos del momento, el entrenamiento de los profesionales sanitarios y la disponibilidad de recursos.

De acuerdo a lo anterior, disponer de índices de medida válidos y fiables, basados en evidencias científicas, garantizará a largo plazo niveles óptimos de calidad en la atención a pacientes que padecen una herida crónica; en el caso de la piel, el principal evaluador de la calidad y el cuidado brindados, es la presencia de lesiones por presión (LPP) (7), permitiendo valorar con precisión los cambios que se presentan, actuar con celeridad y efectividad para disminuir los tiempos de curación y al profesional, le facilita la toma de decisiones acertadas en relación con el tratamiento y prevención. Así mismo, existen en la bibliografía varias escalas que miden el riesgo de presentar una LPP (8) pero, pocas para valorar el proceso de cicatrización; varios autores $(9,10,11,12,13$, $14,15,16,17,18)$ proponen diversas escalas de medida para evaluar este proceso basadas en criterios de calidad.

Estos instrumentos de medida valoran diferentes características de la herida y sus cambios en el tiempo, pero tienen la desventaja de ser difíciles de diligenciar, extensos $y$, en muchos casos solo pueden ser diligenciados por profesionales expertos en el manejo de heridas, lo que hace más difícil la valoración (19).

Adicional a lo anterior no todas las escalas de medida del proceso de cicatrización lo son, algunos son métodos de clasificación de la severidad de las LPP y en la bibliografía no se reflejan aspectos claves para su uso como la fiabilidad, la sensibilidad, la especificidad y el valor predictivo de muchas de ellas, evidenciando problemas importantes, desde el punto de vista metodológico, que interfieren en la interpretación final de los resultados obtenidos disminuyendo la fiabilidad y validez de las mismas (18).

Aunque en Colombia es casi nula la experiencia en este tema, la realidad es que, desde hace algún tiempo, se vienen utilizando instrumentos para valorar el proceso de cicatrización en heridas crónicas (la escala PUSH (20), la reversión del estadio de Shea (21), entre otras), pero hasta el momento no se ha evaluado su uso lo que deja este proceso con vacíos importantes.

En España, en el año 2011, se desarrolló el Índice Resultados Esperados de la Valoración y Evaluación de la Cicatrización de las Heridas Crónicas RESVECH 2.0 (22) con el fin de hacer un consenso entre los profesionales encargados de la atención de los pacientes con heridas crónicas, encontrándose adecuada validez y fiabilidad, convirtiéndolo en una herramienta ideal para ser usado en cualquier 
ámbito y en cualquier tipo de herida crónica.

La valoración que propone el índice de medida RESVECH 2.0 está sustentada en la revisión del conocimiento científico, en el consenso por expertos y en su uso en guías de evidencia y protocolos españoles. Sus características más relevantes son: facilidad en el uso (seis parámetros, la graduación se da de menor a mayor), la descripción exhaustiva de cada una de las categorías de análisis, así como la posibilidad de identificar la característica que impide el avance de la cicatrización; esto, en conjunto, permite la estandarización de cuidados, el aprovechamiento de los recursos, y su evaluación posterior (21).

Por todo lo anterior, se planteó el presente trabajo con el objetivo de estudiar la validez y la fiabilidad del índice RESVECH 2.0 en población colombiana, como una herramienta para valorar el proceso de cicatrización en todo tipo de heridas crónicas.

\section{MATERIALES Y MÉTODOS}

\section{Diseño del estudio}

Estudio metodológico de validación del índice de medida del proceso de cicatrización para todo tipo de heridas crónicas RESVECH 2.0 en el periodo de Junio 2013 - Abril de 2016.

\section{Población estudio}

Se incluyeron pacientes mayores de edad que hacían uso de los servicios de salud de las instituciones participantes con al menos una de las heridas crónicas incluidas en este estudio (Lesiones por Presión (LPP) o úlceras de extremidad inferior (UEI), sin importar el sexo, ni el tratamiento que estuvieran recibiendo.

\section{Calculo de tamaño muestral}

La muestra se estimó según los estándares internacionales de validación de escalas, que oscila entre cinco y veinte pacientes por cada ítem que compone el instrumento (23,
24); dado que el RESVECH 2.0 se compone de seis ítems se estimó una muestra de 120 pacientes, incluyendo las posibles pérdidas durante el estudio; Previamente se realizaron sesiones de entrenamiento por el autor de la escala para la utilización del índice a las enfermeras de las clínicas de heridas y servicios de hospitalización de cada uno de los hospitales incluidos en el estudio, las cuales tenían experiencia comprobada en el manejo de pacientes con heridas.

Los datos se recogieron mediante un instrumento de recolección de datos denominado CRD (Cuaderno de Recogida de Datos, creados solo para este estudio), se realizó un seguimiento a los pacientes cada quince días, durante diez semanas (cinco evaluaciones), durante los cuales se diligenciaron los CRD y el índice RESVECH 2.0 para cada lesión, si el paciente tenía varias lesiones se diligenciaba un CRD diferente por lesión; el CRD contenía aspectos sociodemográficos, y de características específicas de las heridas analizadas.

\section{Análisis estadístico}

\section{El índice de medida RESVECH 2.0}

Este índice contiene seis categorías de medida diseñado para valorar y evaluar el proceso de cicatrización deheridas crónicas, se diligenciaba al principio de cada cuestionario de medida se utilizaron variables básicas de identificación:

» Dimensiones de la úlcera: el largo se tomó de manera céfalocaudal y el ancho perpendicular al largo, (expresados ambos en cms2). Los valores de cada medida se multiplicaron para obtener las dimensiones aproximadas de la herida, pues, los valores pueden variar entre $0 \mathrm{y}>$ 100 cms 2 y los puntajes de la sub-categoría pueden estar en el rango de 0 a 6 que se calificará según el tamaño de la lesión.

» Profundidad/tejidos afectados: varía entre 0 y 4 puntos dependiendo de la lesión, 
comprende: piel intacta o cicatrizada, afectación de la dermis-epidermis, del tejido subcutáneo, del músculo, o de la afectación del hueso y tejidos anexos.

" Bordes: es el tejido que limita el lecho de la herida, se califica de 0 a 4 y comprende: bordes no distinguibles, difusos, delimitados, dañados y engrosados.

» Tipo de tejido en el lecho de la herida: Se refiere a las características del tejido en el lecho de la lesión, si se calificara el peor tejido presente tendríamos: Necrótico-esfacelo, tejido de granulación, tejido epitelial, cerrada- cicatrizada.

» Exudado: este ítem se valoró con el cambio de apósito, de acuerdo con la clasificación de la World Union of Wound Healing Societies (WUWHS) e incluye: apósito seco/cicatrizado, húmedo, mojado, saturado y con fuga de exudado.

» Infección/inflamación: se validó según los signos de infección/inflamación presentes en la herida en una sub-categoría que va de 1 a 14; se calificó 1 presencia de la categoría y 0: cuando estuvo ausente y son: dolor que va en aumento, eritema y edema en la perilesión, incremento de la temperatura y del exudado, exudado purulento, tejido friable y que sangra con facilidad, herida estancada que no progresa, tejido compatible con biofilms, olor, hipergranulación, ampliación del tamaño de la herida, lesiones satélite y palidez del tejido.

Para obtener el puntaje total de la escala se suman los valores de los seis ítems, con un rango de calificación de mínimo 0 y máximo 35 puntos, cuanta más alta es la puntuación la herida se encuentra en más malas condiciones y viceversa, además permite al evaluador darse cuenta en cuál de los ítems evaluados debe colocar más atención y realizar los cambios pertinentes.

Por ser un índice en español no se hizo necesaria la traducción - retrotraducción, pero si se realizaron pruebas de comprensión, tiempo de respuesta, comprensión de las subcategorías de medida, facilidad para calificar el índice entre otras y para eso se realizó una prueba piloto con cinco enfermeras ajenas al estudio expertas en el cuidado de pacientes con heridas cada una evaluó un total de cinco pacientes, los cuales fueron evaluados al azar por el grupo de enfermeras en dos ocasiones diferentes para comparar puntajes, obteniendo resultados similares en todos los casos, esta prueba se conoce como test- retest consiste en la aplicación del mismo instrumento a una misma muestra de sujetos en dos o más ocasiones, bajo condiciones similares, estos resultados se analizaron utilizando el Coeficiente de correlación de Pearson, el cual muestra hasta donde los resultados obtenidos pueden ser generalizados a través del tiempo.

Para realizar las pruebas de validez del índice de medida RESVECH 2.0, se contó con el grupo de enfermeras de cada institución (ocho en total), con las cuales se realizaron dos talleres donde se les explico el índice y como diligenciarlo, además se realizaron ejercicios de valoración para aclarar las posibles dudas, seguidamente se realizó la validez de contenido utilizando veinte pacientes por ítem evaluado es decir 120 pacientes ( el índice RESVECH cuenta con seis ítems), en esta fase de la validación se evaluó que cada categoría del índice si "evaluara lo que pretendía evaluar" teniendo en cuenta la capacitación previa a las enfermeras; es de anotar también que para realizar el análisis factorial y analizar la relación entre las variables se utilizó la medida de la adecuación muestral de Kaiser-Meyer-Olkin que contrasta si las correlaciones parciales entre las variables son pequeñas y la prueba de esfericidad de Bartlett que contrasta si la matriz de correlaciones es 
una matriz de identidad, que indicaría que el modelo factorial es inadecuado.

Otra de las pruebas realizadas al índice y tal vez la de mayor importancia es la de sensibilidad al cambio en la cual se realizó un modelo de medidas repetidas con factor tiempo (ANOVA de medidas repetidas).

Este modelo se analizó de manera general, comparándolo por etiología, cicatrización y en función de las mejores y peores puntuaciones al inicio (mediante al cálculo de los cuartiles), además para medir la consistencia interna se utilizó el coeficiente alfa de Cronbach modificado ya que el índice RESVECH tiene variación en las valoraciones de cada categoría y no se calificaban todas de la misma manera fue necesario medirlo en dos momentos, tanto al inicio del estudio como en cada momento de medida. Este coeficiente también fue medido en función del tipo de herida, para ver si había diferencias, en función de este parámetro.

\section{RESULTADOS}

\section{Características de la población y de las heridas analizadas.}

Para este estudio la muestra fue de 120 pacientes (una lesión por paciente), todos con una edad promedio de edades entre los 62 y los 86 años (desviación estándar [DE], 75.2+/17.2). El tiempo de administración del índice osciló entre 3 y 6 min, con un tiempo promedio de 5+/-2 min. No hubo ninguna complicación en la aplicación del índice RESVECH 2.0, ni tampoco la necesidad de ajustarlo. El $46 \%$ de las lesiones analizadas contaba con una extensión entre 19 y 42 cms2, el 37,2\% entre 0 y $18 \mathrm{cms} 2,13,8 \%$ mayor de $36 \mathrm{cms} 2$, y solo una lesión sobrepasaba los 100 cms2; En cuanto a la antigüedad de la herida, el tiempo promedio fue 3,4 meses $\pm 2,4$ meses (me $=3$ meses) y rango de 1 a 16 meses. En cuanto al tipo de heridas, se encontró que $65 \%$ eran LPP, y $35 \%$ correspondían a UEI (de las cuales 38\% eran úlceras venosas, 19\% úlceras (UI) y 43\% úlceras neuropáticas).

En cuanto al sexo de los participantes 69 eran mujeres $(83,3 \%)$, casados $55(66.6 \%)$, nivel de escolaridad primaria $77(64.3 \%)$ secundaria $15(12.3 \%)$ y posgrado 6(6.6\%), al analizar el estrato socioeconómico 70 (46,6\%) son de estrato 1 y 2, 30 (25\%) pertenece al estrato 4 y 5 no tuvimos pacientes de estrato 3 , en cuanto al tratamiento no se tuvo en cuenta por la variabilidad del mismo y por qué no era un objetivo del estudio como tal, además cada profesional era libre de indicar el tratamiento que mejor le pareciera de acuerdo a las necesidades del paciente, el grupo investigador no influyo en el mismo, la mayoría de los pacientes pertenecían al régimen subsidiado, solo 10 pacientes eran de índole particular.

Al analizar la profundidad de las lesiones se encontró que $64,3 \%$ de ellas se extendía hasta el tejido subcutáneo sin llegar a la fascia del músculo, $42 \%$ afectaba el músculo y solo $6.1 \%$ se extendía hasta el hueso y los tejidos anexos (como tendones, ligamentos, cápsula articular etc.).

En cuanto a los bordes de las heridas analizadas, $66 \%$ los tenía delimitados, $31 \%(n=26)$ dañados y una pequeña porción los tenia engrosados o "envejecidos". En esta misma línea, del total de lesiones, 55,6\% estaban maceradas y $13,5 \%$ tenía algún tipo de tunelización o cavitación.

El principal tejido presente en el lecho de la herida era el necrótico o con esfacelos $66 \%$, el $38.6 \%$ tejido de granulación, $2,6 \%(n=3)$ tenía tejido necrótico y solo $1,4 \%$ de las 84 lesiones analizadas tenia tejido epitelial o estaba en la última fase del proceso de cicatrización.

En el momento de la curación se valoró la presencia de exudado en el apósito que tenía la herida, según las recomendaciones de la World Union of Wound Healing Societies (WUWHS) 
para este fin y se encontró que $68 \%$ tenía el apósito saturado de exudado, 25.2\% estaba mojado y en $8,5 \%$ se observó una fuga de exudado o simplemente estaba seco, es decir, sin presencia alguna de exudado, pero no estaba cicatrizada.

Al calcular el alfa de Cronbach para el índice RESVECH 2.0 se obtiene una alfa un alfa de Cronbach basado en los elementos tipificados de 0,74 , con valores estables, las correlaciones elemento-total se mantuvieron por encima de 0,36 . Al observar los valores del alfa basado en los elementos tipificados, estos aumentan cuando se acerca a las medidas finales, ya que si una lesión disminuye su puntuación los valores de los elementos de la escala se van pareciendo más (son más consistentes) y se obtienen valores por encima de 0.96 .

La validez de contenido como se mencionó anteriormente se realizó por medio de la prueba de esfericidad de Barlet, con resultados de $\mathrm{X} 2=$ 84.020, grados de libertad de 14 y $p$ valor de 0.001y la prueba de adecuación de la muestra de $\mathrm{KMO}$ fue de 0.623. Así mismo al realizar las pruebas de confiabilidad prueba test re-test El coeficiente de correlación y concordancia de Lin fue 0.91 (IC 95\%: 0.87 - 0.95).

\section{DISCUSIÓN}

Para evaluar el índice RESVECH 2.0 se ha estudiado la fiabilidad inter observador, como un instrumento "a pie de cama" que será utilizado por un gran número de profesionales encargados del cuidado de pacientes con heridas crónicas.

Aunque, es difícil comparar con estudios de validación de otros instrumentos de medida sobre todo, porque la técnica para realizar, establecer y evaluar la fiabilidad del índice es diferente a la utilizada en otros estudios, así como los procedimientos estadísticos que se usan; además de utilizar el alfa de Cronbach, medida muy útil en la psicometría y que muestra importantes re sultados cuando se usa en este tipo de investigaciones (20). Sumado a lo anterior, en la actualidad este índice es utilizado de forma amplia en países como España donde ya aparece en sus guías de práctica clínica y protocolos de manejo de heridas, y a la vez está siendo validado en diferentes países de habla hispana.

Una característica importante de este estudio es que se pudo realizar la evaluación de la validez de criterio de forma adecuada, gracias a que el número de evaluaciones hechas a los pacientes fueron las apropiadas como se hizo en otros estudios de validación $(26,27,28,29)$, lo que ayuda a mejorar los resultados clinicométricos del índice RESVECH 2.0 en la población colombiana y aporta características de medida muy fuertes en la valoración de heridas crónicas, lo que hace que este índice se convierta es una poderosa herramienta de valoración en ámbitos clínicos y comunitarios, permitiéndole al profesional identificar en poco tiempo las dificultades que presenta la herida para cicatrizar.

El índice "RESVECH V2.0. "Resultados esperados de la valoración y evolución de la cicatrización de las heridas crónicas" se constituye en nuestro país como el primer instrumento de valoración y evaluación del proceso de cicatrización, aplicado a todo tipo de heridas crónicas, independiente de su etiología y se convierte en una herramienta que puede ser utilizada sin importar el tamaño de la lesión hasta el fin de la cicatrización (29).

Como limitaciones del estudio se puede mencionar el tiempo de seguimiento de los participantes que pudo haber sido un poco mayor para visualiza rasí mejor los diferentes puntajes de las heridas desde el inicio hasta su cierre definitivo.

\section{CONCLUSIONES}

El análisis cuantitativo del cuestionario pone 
en evidencia que el índice RESVECH 2.0 para medir el proceso de cicatrización de las heridas presenta valores de fiabilidad y validez adecuados para usarlo en la práctica clínica.

Este estudio se convierte en una referencia a nivel nacional en la valoración de heridas crónicas ya que como se mencionó al principio del estudio son pocos los instrumentos con los que cuenta enfermería para la valoración del paciente con heridas, además de que permite integrar los planes de cuidado a la valoración obtenida, arroja datos de mucha importancia y permite al profesional tomar decisiones rápidas basadas en la evidencia disponible, ya que los valores obtenidos en la evaluación de la sensibilidad permite observar los cambios por pequeños que estos sean en poco tiempo.

Se recomienda seguir realizando diferentes estudios de validación con este índice de medida, utilizando las técnicas investigativas adecuadas, además el utilizar escalas, índices o instrumentos de medida, le permite menor tiempo de curación, menor estancia hospitalaria y mejor calidad de vida para el paciente.

\section{CONFLICTO DE INTERÉS}

El autor declara no tener conflictos de interés.

\section{REFERENCIAS}

1. Ministerio de Sanidad y Consumo. Plan de Calidad para el Sistema Nacional de Salud. Madrid; 2006.

2. Gutiérrez FF. Prevenir las úlceras por presión es garantizar la calidad asistencial. Enfermería Científica. 1993; 140: 7-10.

3. Soldevilla AJ, Torra i Bou JE, Verdú SJ, López CP. 3.er Estudio Nacional de Prevalencia de Úlceras por Presión en España, 2009: Epidemiología y variables definitorias de las lesiones y pacientes.
Gerokomos. 2011 [citado 2014 Jun 20]; 22(2):77-90.Disponible en: http://scielo. isciii.es/scielo.php?script=sci_arttext\&pi$\mathrm{d}=$ S1 134-928X2011000200005\&Ing=es. http://dx.doi.org/10.4321/S1 134 928X2011000200005.

4. Bergstrom N, Braden B. A prospective study of pressure sore risk among institutionalized elderly. JAGS. 1992; 40 (8): 747-758.

5. Edwards M. The rationale for the use of risk calculators in pressure sore prevention, and the evidence of the reliability and validity of published scales. J Advan Nursing 1994; 20: 288-296.

6. Fagan R, Closs SJ. Pressure sore management in the independent sector: 1 . Br J Nursing 1996; 5 (18): 1138-1145.

7. Barbero FJ, Villar R. Mejora continua de la calidad en las lesiones por presión mediante la valoración del riesgo de aparición. Enfermería Clínica 1998; 8 (4): 141-150.

8. García FF, Pancorbo HP, Soldevilla AJ, Blasco García C. Escalas de valoración del riesgo de desarrollar úlceras por presión. Gerokomos. 2008 Sep [citado 2014 Jun 24]; 19(3): 136-144. Disponible en:http:// scielo.isciii.es/scielo.php?script=sci_arttext\&pid=S1134928X2008000300005\&lng=es.http://dx.doi.org/10.4321/S1134928X2008000300005.

9. Bates JB, Vredevoe DL, Brecht ML. Validity and reliability of the Pressure Sore Status Tool. Decubitus. 1992; 11(5), 20-28.

10. Emperanza JL, Aranegui $\mathrm{P}$, Ruiz M et. al. A simple Severity index for pressure ulcers. Journal of Wound Care, 2000; 9(2), 86-90.

11. Falanga V, Saap LJ, Ozonoff A. Wound bed score and its correlation with healing 
of chronic wounds. Dermatol Ther. 2006; 19, 383-390.

12. Gardner S, Frantz R, Bergquist S, Shin Ch. A Prospective Study of the Pressure Ulcer Scale for Healing (PUSH). Journal of Gerontology: Medical Sciences. 2005; 60(1), 93-97

13. Gûnes U. A Prospective Study Evaluating the Pressure Ulcer Scale for Healing to Assess Stage II, Stage III, and Stage IV Pressure Ulcers. OWM, 55(5), 48-52.

14. Maklebust J, Rodeheaver G, Bartolucci A, et al. Pressure Ulcer Scale for Healing: Derivation and Validation of the PUSH Tool. ADV WOUND CARE. 1997; 10(5).

15. National Pressure Ulcer Advisory Panel and European Pressure Ulcer Advisory Panel (NPUAP-EPUAP). Prevention and treatment of pressure ulcer: clinical practice guideline. Washington DC: NPUAP; 2009.

16. Ratliff CR, Rodeheaver GT. Use of the $\mathrm{PUSH}$ tool to measure venous ulcer healing. Ostomy Wound Management. 2005; 51(5), 58-63.

17. Sanada H, Moriguchi T, Mlyachi $Y$, et al. Reliability and validity of DESIGN, a tool that classifies pressure ulcer severity and monitors healing. JWC. 2004; 13(1), 1318.

18. Maklebust J, Rodeheaver G, Bartolucci A et al. Pressure Ulcer Scale for Healing: Derivation and Validation of the PUSH Tool. Adv Wound Care 1997; 10 (5): 96-101.

19. Shea DJ. Pressure Sores Classification and Management. Clin Orthop Rel Res 1995; 112: 89-100.
20. Restrepo MJ, Verdú SJ. Desarrollo de un índice de medida de la evolución hacia la cicatrización de las heridas crónicas. Gerokomos.Dic [citado 2014 Jun 24]. 2011; 22(4): 176-183. Disponible en: http:// scielo.isciii.es/scielo.php?script=sci_arttext\&pid=S1134-928X2011000400005\&l ng=es. http://dx.doi.org/10.4321/S1134 928X2011000400005.

21. Polit DF, Hungler BP. Investigación científica en Ciencias de la Salud: principios y métodos. 6a. ed. México: McGraw-Hill Interamericana. 2000; pp. 398-401.

22. Argimon Pallás JM, Jiménez Vila J. Métodos de investigación clínica y epidemiológica: Ediciones Harcourt; 2006.

23. World Union of Wound Healing Societies (WUWHS). Principios de las mejores prácticas: Exudado en las heridas y utilidad de los apósitos. Un documento de consenso. London: MEPLtd: WUWHS; 2007.

24. Deeks JJ. Pressure sore prevention: using and evaluating risk assessment tools. $\mathrm{Br} \mathrm{J}$ Nursing 1996; 585: 313-320.

25. Braden BJ, Bergstrom N. Predictive validity of the Braden scale for pressure sore risk in a nursing home population. Res Nurs Health 1994; 17: 459-470.

26. Watkinson C. Inter-rater reliability of risk-assessment scales. Professional Nurse 1996; 2 (2): 751-756.

27. Ramundo JM. Reliability and validity of the Braden scale in the home care setting. JWOCN.1995 Mayo; 22 (3): 128-34. 